\title{
Heart rate variability in children with aortic valve stenosis - a pilot study
}

Bozena Werner ${ }^{1}$, Anna Piorecka-Makula', Waldemar Bobkowski²

1 Medical University of Warsaw, Poland

2Poznan University of Medical Sciences, Poland

Submitted: 9 January 2012

Accepted: 16 October 2012

Arch Med Sci 2013; 9, 3: 535-539

DOI: $10.5114 /$ aoms.2013.34880

Copyright $\odot 2013$ Termedia \& Banach

\section{Abstract}

Introduction: The aim of our prospective study was to evaluate heart rate variability (HRV) in children with aortic valve stenosis (AS) and its relationship with left ventricular mass and peak transaortic valve pressure gradient (PG).

Material and methods: Sixty children with AS divided into 3 groups according to their PG and 60 healthy controls were studied. Holter ECG monitoring with time domain HRV analysis was performed. Left ventricular mass was calculated by echocardiography.

Results: Mean values of all HRV parameters were statistically significantly lower $(p<0.001)$ in children with AS than in controls (respectively: SDNN 127.8 $\pm 28.2 \mathrm{~ms} ; 162.6 \pm 38.0 \mathrm{~ms}$, SDNN day $99.7 \pm 26.6 \mathrm{~ms} ; 134.1 \pm 36.1 \mathrm{~ms}$, SDNN night $99.9 \pm 32.8 \mathrm{~ms} ; 123.4 \pm 45.7 \mathrm{~ms}$, SDANN $112.2 \pm 27.7 \mathrm{~ms} ; 142.4 \pm 34.6$, SDNNi 62.2 $\pm 16.2 \mathrm{~ms} ; 75.9 \pm 21.6$, RMSSD $39.6 \pm 12.1 \mathrm{~ms} ; 50.3 \pm 16.7 \mathrm{~ms}$, rMSSD day $33.6 \pm 10.9$ ms; $43.1 \pm 14.7 \mathrm{~ms}$, rMSSD night $49.8 \pm 18.1 \mathrm{~ms} ; 64.4 \pm 24.9 \mathrm{~ms}$, pNN50 $16.4 \pm 9.5 \%$; $23.5 \pm 11.7 \%$, pNN50 day $12.0 \pm 8.5 \%$; $18.4 \pm 10.7 \%$, pNN50 night; $26.5 \pm 14.8 \%$; $36.4 \pm 17.4 \%$. No significant differences between the mean values of HRV parameters in children with different PG and with and without myocardial hypertrophy were found. In children with AS and ventricular arrhythmia SDNN day was significantly lower $(p<0.05)$ compared to patients without arrhythmia (94.9 $\pm 22.1 \mathrm{~ms}$ vs. $109.3 \pm 22.5 \mathrm{~ms}$ ).

Conclusions: In children with AS the balance of the autonomic nervous systemic disturbed which manifests in an increase in sympathetic and decrease in parasympathetic activity. Transaortic valve pressure gradient and myocardial hypertrophy do not influence the HRV. The SDNN reduction during the day period may indicate the risk of ventricular arrhythmia in children with AS.

Key words: heart rate variability, aortic stenosis, children.

\section{Introduction}

Aortic valve stenosis (AS) is an important clinical problem in children who are at risk of increased ventricular arrhythmia and sudden cardiac death.

The dysfunction of the autonomic nervous system influences the increased and/or heterogeneous repolarization of the cardiac myocytes, which in turn contributes to the occurrence of ventricular arrhythmia in the re-entry and triggered activity mechanisms. The evaluation of heart rate variability (HRV) is a non-invasive method of determining the function of the autonomic nervous system [1-3].

The assessment of HRV in children with valvular aortic stenosis has not been studied so far and no articles concerning this topic were found in the literature.

\author{
Corresponding author: \\ Prof. Bozena Werner MD, PhD \\ Department of Pediatric \\ Cardiology \\ and General Pediatrics \\ Medical University of Warsaw \\ 24 Marszalkowska St \\ 00-576 Warsaw, Poland \\ Phone: +48 226298317 \\ E-mail: \\ bozena.werner@wum.edu.pl
}


The aim of our prospective study was to evaluate HRV in children with AS.

\section{Material and methods}

Sixty children with AS aged 5 to 18 years old (mean: $12.78 \pm 3.73$ years, 16 girls and 44 boys) were included in the study. They were divided into three groups, depending on the peak systolic pressure gradient between the left ventricle and the aorta (PG), calculated using Doppler echocardiography. Group I consisted of 21 children with mild aortic valve stenosis (PG 25-39 mm Hg), group II comprised 27 children with moderate aortic valve stenosis (PG 40-69 $\mathrm{mm} \mathrm{Hg}$ ), and group III included 12 children with severe aortic valve stenosis (PG $\geq 70 \mathrm{~mm} \mathrm{Hg}$ ). The control group (C) comprised 60 healthy, age- and BSA- (body surface area) matched children.

In all children 2D echocardiography was performed and the left ventricular mass (Devereux formula) and left ventricular mass index (LVMI = LVM/BSA) were calculated. Left ventricular hypertrophy was defined as an LVMI that was over the $95^{\text {th }}$ percentile, i.e. LVMI $\geq 84 \mathrm{~g} / \mathrm{m}^{2}$ for girls and $\mathrm{LVMI} \geq 103 \mathrm{~g} / \mathrm{m}^{2}$ for boys. 24-hour ECG Holter monitoring (Cardioscan 8, MTM-multitechmed, 128 samples per second) was used to scan for arrhythmias and time-domain heart rate variability parameters. The following parameters were analysed for a 24 hour period: SDNN (the standard deviation of all filtered R-R intervals over the length of the analysis), SDANN (the standard deviation of the means of all filtered R-R intervals for all 5-minute segments of the analysis), SDNNi (the mean of the standard deviations of all filtered R-R intervals for all 5-minute segments of the analysis), rMSSD (the square root of the mean of the sum of squares of differences between adjacent filtered R-R intervals over the

Table I. Mean values of time-domain HRV in children with AS and healthy children

\begin{tabular}{|lccc|}
\hline Parameter & $\begin{array}{c}\text { Children } \\
\text { with AS }\end{array}$ & $\begin{array}{c}\text { Control (C) } \\
\text { group }\end{array}$ & $\begin{array}{c}\text { Value } \\
\text { of } p\end{array}$ \\
\hline SDNN [ms] & $127.8 \pm 28.2$ & $162.6 \pm 38.0$ & $<0.0001$ \\
\hline SDNN day [ms] & $99.7 \pm 26.6$ & $134.1 \pm 36.1$ & $<0.0001$ \\
\hline SDNN night [ms] & $99.9 \pm 32.8$ & $123.4 \pm 45.7$ & $<0.0005$ \\
\hline SDANN [ms] & $112.2 \pm 27.7$ & $142.4 \pm 34.6$ & $<0.0001$ \\
\hline SDNNi [ms] & $62.2 \pm 16.2$ & $75.9 \pm 21.6$ & $<0.0001$ \\
\hline RMSSD [ms] & $39.6 \pm 12.1$ & $50.3 \pm 16.7$ & $<0.0001$ \\
\hline rMSSD day [ms] & $33.6 \pm 10.9$ & $43.1 \pm 14.7$ & $<0.0001$ \\
\hline rMSSD night [ms] & $49.8 \pm 18.1$ & $64.4 \pm 24.9$ & $<0.001$ \\
\hline pNN50 [\%] & $16.4 \pm 9.5$ & $23.5 \pm 11.7$ & $<0.0005$ \\
\hline pNN50 day [\%] & $12.0 \pm 8.5$ & $18.4 \pm 10.7$ & $<0.0001$ \\
\hline pNN50 night [\%] & $26.5 \pm 14.8$ & $36.4 \pm 17.4$ & $<0.005$ \\
\hline
\end{tabular}

length of the analysis), and pNN50 (the percentage of differences between adjacent filtered R-R intervals that are greater than $50 \mathrm{~ms}$ for the whole analysis). Moreover, day (12 noon - 4 PM) and night (12 midnight - 4 AM) values of SDNN, rMSSD and pNN50 were assessed.

The approval from the Institutional Bioethics Committee for the study project according to the above presented protocol and informed consent from all parents and children above 16 years old were obtained.

\section{Statistical analysis}

Statistical analysis was performed using SPSS/PC + software.

The Shapiro-Wilk test was performed to evaluate whether the distribution of the variables was normal. Continuous variables were described using localisation and dispersion statistics, so that the mean and standard deviation values were obtained.

Due to the homogeneity of the group in terms of age, the significance of the differences between mean parameter values in the study group in dependence on the transvalvular pressure gradient was tested using variance analysis (ANOVA). The differences between the mean parameter values in the study group in dependence on the presence of arrhythmia were analysed using covariance analysis (ANCOVA) with age as a covariate (due to the significantly higher age of children with arrhythmia in comparison to children without rhythm disturbances). The values of studied parameters were corrected according to age.

The significance of the differences between the mean values observed in the study and control (age- and BSA-matched) groups was analysed using Student's $t$-test. Similarly, Student's $t$-test was used to analyse the differences between mean values observed in the subgroups of the study groups, defined based on the gradient level and arrhythmia and age- and BSA-matched subgroups of the controls. Correlations between HRV parameters and PG were analysed using Pearson correlation coefficient.

The significance level for the verification of hypotheses was set at $p<0.05$. All analysed statistical tests were bilateral.

\section{Results}

The mean values of the time-domain heart rate variability obtained in children with AS and healthy age- and BSA-matched volunteers are presented in Table I.

Mean values of all studied HRV parameters were statistically significantly lower $(p<0.001)$ in children with AS than the respective parameters in controls.

Table II presents the comparative analysis of mean values of the analysed HRV parameters in particular groups of studied children. 
Table II. Mean values of HRV parameters in three groups of children with AS according to the pressure gradient between the left ventricle and the aorta

\begin{tabular}{|lcccc|}
\hline HRV parameter & Group I & Group II & Group III & Value of $p$ \\
\hline SDNN [ms] & $133.3 \pm 31.5$ & $125.4 \pm 26.7$ & $123.4 \pm 26.2$ & NS \\
\hline SDNN day [ms] & $103.1 \pm 29.3$ & $94.7 \pm 22.8$ & $105.1 \pm 29.7$ & NS \\
\hline SDNN night [ms] & $106.2 \pm 40.3$ & $97.3 \pm 29.1$ & $94.7 \pm 26.4$ & NS \\
\hline SDANN [ms] & $120.1 \pm 30.7$ & $110.6 \pm 26.0$ & $101.8 \pm 23.5$ & NS \\
\hline SDNNi [ms] & $61.8 \pm 15.8$ & $60.4 \pm 15.2$ & $67.2 \pm 19.3$ & NS \\
\hline rMSSD [ms] & $41.4 \pm 12.8$ & $36.1 \pm 9.4$ & $44.2 \pm 14.8$ & NS \\
\hline rMSSD day [ms] & $35.0 \pm 10.5$ & $30.2 \pm 8.6$ & $38.4 \pm 14.6$ & NS \\
\hline rMSSD night [ms] & $51.9 \pm 20.5$ & $46.4 \pm 15.1$ & $53.9 \pm 19.9$ & NS \\
\hline pNN50 [\%] & $17.7 \pm 9.9$ & $13.7 \pm 7.7$ & $20.0 \pm 11.3$ & NS \\
\hline pNN50 day [\%] & $13.0 \pm 8.3$ & $9.5 \pm 6.9$ & $15.6 \pm 10.9$ & NS \\
\hline pNN50 night [\%] & $29.2 \pm 16.2$ & $23.1 \pm 12.7$ & $29.3 \pm 16.3$ & NS \\
\hline
\end{tabular}

Table III. Correlations between parameters of HRV and pressure gradient

\begin{tabular}{|c|c|c|c|c|c|c|c|c|c|c|c|}
\hline & SDNN & SDNNday & SDNN night & SDANN & SDNNI & rMSSD & rMSSD d & rMSSD n & pNN50 & pNN50 d & pNN50 n \\
\hline PG & -0.05 & 0.09 & -0.05 & -0.18 & 0.19 & 0.10 & 0.13 & 0.06 & 0.10 & 0.12 & 0.02 \\
\hline
\end{tabular}

Table IV. Mean values of HRV parameters in children with AS with and without myocardial hypertrophy

\begin{tabular}{|lccc|}
\hline Parameter & $\begin{array}{c}\text { Children with } \\
\text { LVMI }>95 \mathrm{c}\end{array}$ & $\begin{array}{c}\text { Children with } \\
\text { LVMI }>95 \mathrm{c}\end{array}$ & $\begin{array}{c}\text { Value } \\
\text { of } p\end{array}$ \\
\hline SDNN [ms] & $132.1 \pm 26.3$ & $126.0 \pm 25.6$ & NS \\
\hline SDNN day [ms] & $104.5 \pm 23.6$ & $97.8 \pm 23.0$ & NS \\
\hline SDNN night [ms] & $98.2 \pm 30.5$ & $100.5 \pm 29.7$ & NS \\
\hline SDANN [ms] & $113.4 \pm 27.9$ & $111.7 \pm 27.1$ & NS \\
\hline SDNNi [ms] & $66.5 \pm 14.8$ & $60.5 \pm 14.4$ & NS \\
\hline RMSSD [ms] & $42.3 \pm 12.4$ & $38.5 \pm 12.0$ & NS \\
\hline rMSSD day [ms] & $35.3 \pm 11.0$ & $32.8 \pm 10.7$ & NS \\
\hline rMSSD night [ms] & $53.9 \pm 19.0$ & $48.2 \pm 18.5$ & NS \\
\hline pNN50 [\%] & $18.5 \pm 9.6$ & $15.5 \pm 9.4$ & NS \\
\hline pNN50 day [\%] & $13.4 \pm 8.7$ & $11.4 \pm 8.3$ & NS \\
\hline pNN50 night [\%] & $29.1 \pm 15.1$ & $25.4 \pm 15.1$ & NS \\
\hline
\end{tabular}

No statistically significant differences were observed between groups I and II, I and III, and II and III, in respect of mean values of all analysed HRV parameters. No correlations were found between the HRV parameters and pressure gradient (Table III).

In 17 children (28.33\%) with AS, LVMI > 95th percentile was observed (range 92.53 to $159.25 \mathrm{~g} / \mathrm{m}^{2}$, mean $\left.115 \pm 19.0 \mathrm{~g} / \mathrm{m}^{2}\right)$. In the remaining group of children with AS, mean LVMI value was 66.4 $\pm 15.7 \mathrm{~g} / \mathrm{m}^{2}$. The mean values of HRV parameters obtained in children with myocardial hypertrophy in the course of valvular aortic stenosis and in chil-
Table V. Mean values of HRV parameters in children with AS with and without arrhythmia

\begin{tabular}{|lccc|}
\hline $\begin{array}{l}\text { HRV } \\
\text { parameter }\end{array}$ & $\begin{array}{c}\text { Children with } \\
\text { AS with } \\
\text { arrhythmia }\end{array}$ & $\begin{array}{c}\text { Children with } \\
\text { AS without }\end{array}$ & $\begin{array}{c}\text { Value } \\
\text { of } p\end{array}$ \\
\hline SDNN [ms] & $125.4 \pm 26.1$ & $128.9 \pm 25.7$ & NS \\
\hline SDNN day [ms] & $94.9 \pm 22.1$ & $109.3 \pm 22.5$ & 0.0248 \\
\hline SDNN night [ms] & $99.6 \pm 30.1$ & $100.0 \pm 29.7$ & NS \\
\hline SDANN [ms] & $103.5 \pm 26.8$ & $116.5 \pm 26.4$ & NS \\
\hline SDNNi [ms] & $66.9 \pm 14.5$ & $59.9 \pm 14.2$ & NS \\
\hline rMSSD [ms] & $41.9 \pm 13.7$ & $38.5 \pm 11.2$ & NS \\
\hline rMSSD day [ms] & $36.9 \pm 10.8$ & $31.9 \pm 10.1$ & NS \\
\hline rMSSD night [ms] & $48.9 \pm 17.9$ & $50.3 \pm 18.4$ & NS \\
\hline pNN50 [\%] & $18.4 \pm 10.4$ & $15.4 \pm 8.9$ & NS \\
\hline pNN50 day [\%] & $14.3 \pm 8.3$ & $10.8 \pm 8.2$ & NS \\
\hline pNN50 night [\%] & $26.0 \pm 16.0$ & $26.7 \pm 14.4$ & NS \\
\hline
\end{tabular}

dren with AS and LVMI below the 95th percentile are presented in Table IV.

The analysis did not demonstrate statistically significant differences between the mean values of HRV parameters in children with and without myocardial hypertrophy in the course of AS.

Ventricular arrhythmias were observed in 20 children $(33.33 \%)$ with AS. In 4 children (19\%) of group I single ectopic ventricular beats were present. Seven patients (26\%) of group II demonstrated not only single ectopic ventricular beats, but couplets of ventricular ectopic beats in one child. Single ventricular beats were observed in 9 children (75\%) 
of group III, and in 3 of them couplets and runs of ectopic beats were also found. No arrhythmias were seen in the control group.

Table $\mathrm{V}$ presents mean values of the analysed HRV parameters corrected according to age in children with AS and arrhythmia and in children with AS and no record of arrhythmia.

In children with AS and ventricular arrhythmia the tendency of lower mean values of the following HRV parameters was observed, compared to children with AS and without arrhythmia: SDNN, SDNN day, SDNN night, SDANN, rMSSD night and pNN50 night. A statistically significant difference was found for SDNN day only.

\section{Discussion}

The interest of cardiologists in the heart rate variability was initiated by Wolf [4], who studied 176 patients with acute myocardial infarction and stated that a decrease in HRV is associated with poor prognosis. According to the published study results, patients with heart failure, ischaemic heart disease, myocardial infarction, hypertension, diabetes, psychiatric disorders, neonatal diseases and low parameters of parasympathetic nervous system activity present a risk of cardiac death [4-7]. There are still only a few reports concerning the significance of HRV parameters in children with heart defects. Buchhorn et al. [8] observed changes in the tone of the autonomic nervous system in the form of HRV decrease in children with heart failure in the course of heart defects. Massin et al. [9] on the other hand reported changes in the tone of the autonomic nervous system in the form of HRV parameter decreases in children with heart defects without concomitant heart failure. In contrast, Heragu and Scott [10] studied 36 children with cyanotic and shunt-dependent heart defects and were not able to point out significant differences in the parameters of time-domain HRV in comparison to healthy children. Kucharska et al. [11] did not observe significant reduction of HRV in children with ventricular septal defect, but noted significantly lower values of the parameters directly dependent on the vagus nerve tone in children with atrial septal defect. Bobkowski et al. [12] observed an increase in the sympathetic system tone in children with mitral valve prolapse.

In our study all parameters of the time-domain HRV were statistically significantly lower in children with valvular aortic stenosis in comparison to the values obtained in healthy children. It indicates that the parasympathetic activity decreases and the sympathetic activity increases in children with AS. In one of the few papers concerning HRV in adults with AS, Jung et al. [13] observed relatively low values of the parameters of the time-domain HRV analysis, and the lowest values in patients with the most impaired exercise tolerance. Stein et al. [14] indicate the significance of HRV evaluation in the prediction of the clinical course of heart defects in adults. Many authors emphasise that the most significant independent risk factor for a cardiac incident is a decreased SDNN value. It was confirmed by the results of studies on patients with cardiomyopathy, myocardial infarction or heart failure [6, 7, 15-18]. Karcz and Ruzyllo [18] consider SDNN to be a significant risk factor for a cardiac incident in patients with cardiomyopathy, although they hold that it is not possible to establish the SDNN threshold value which would differentiate the prognosis. According to Kleiger et al. [6] an SDNN value below $100 \mathrm{~ms}$ in patients after myocardial infarction indicates poor, and below 50 ms very poor prognosis. Nolan et al. [7] consider SDNN as the best independent risk factor and the most sensitive parameter for cardiac incidents in patients with heart failure, whereas short-term variability parameters, such as rMSSD and pNN50, have no prognostic value.

No statistically significant difference between the values of HRV parameters in the three groups of studied children with AS was observed in our study. Our observations are in concordance with the data published by Jung et al. [13], who used correlation analysis and did not demonstrate an influence of transvalvular pressure gradient on HRV in adults with AS.

The views concerning the association between HRV and myocardial hypertrophy are divergent. According to some authors, a decrease in HRV is associated with heart hypertrophy regardless of its aetiology $[16,19]$. Others, on the other hand, did not prove such a relationship [20-22]. The results of our own study indicate no statistically significant differences between HRV parameters in children with and without myocardial hypertrophy in the course of valvular aortic stenosis. There are no reports on HRV in children with myocardial hypertrophy in the course of AS and the published results in adults are inconsistent. Alter et al. [16] report a decrease of HRV parameters, mainly SDNN, in adults with myocardial hypertrophy secondary to AS, while Jung et al. [13] did not observe a correlation between HRV and left ventricular mass in adults with AS. The most prevalent view in the literature is that a disturbance in the sympathetic-parasympathetic balance, manifesting as an increase of the sympathetic activity, increases the incidence of complex ventricular arrhythmias and reduces the trigger threshold for ventricular fibrillation [6, 12, 15, 23]. Myerburg [24] suggests that the rapid increase in sympathetic tone in young people without organic heart disease may be a predisposing factor for idiopathic ventricular tachycardia. The degree to which this balance of both types of autonomic modulation is 
disturbed in favour of a sympathetic response is significantly associated with higher incidence of ventricular arrhythmia in children with mitral valve prolapse [12].

In our study SDNN day was the only parameter that showed a statistically significant difference between patients with AS and with arrhythmia and those without arrhythmia, which indicates the increase in sympathetic tone during the day period. Fauchier et al. [15] stated that the SDNN value is an independent, significant risk factor for an arrhythmic incident in patients with cardiomyopathy. According to the literature review, most of the authors believe that the parameters of total and long-term heart rate variability, i.e. SDNN and SDANN, have the highest prognostic value, among all time-domain analysis parameters $[6,14,18]$. Especially disadvantageous is the maintenance of decreased HRV values, considered to be a negative prognostic factor, and the treatment may improve the HRV $[10,17,25]$.

Due to the small number of patients with AS and significant arrhythmia, the role of HRV in this aspect needs further research.

In conclusion, the balance of the autonomic nervous system is disturbed in children with valvular aortic stenosis, which manifests in an increase in sympathetic and decrease in parasympathetic activity. Neither the transvalvular gradient nor myocardial hypertrophy influences the heart rate variability in children with aortic valve stenosis. SDNN reduction during the day period may indicate a risk of ventricular arrhythmia in children with aortic valve stenosis.

\section{References}

1. Malik M, Bigger TJ, Camm JA, et al. Heart rate variability. Standards of measurement, physiological interpretation and clinical use. Task Force of the European Society of Cardiology and the North American Society of Pacing and Electrophysiology. Guidelines. Eur Heart J 1996; 17: 354-81.

2. Heitmann A, Huebner T, Schroeder R, et al. Multivariate short-term heart rate variability: a pre-diagnostic tool for screening heart disease. Med Biol Eng Comput 2011; 49: 41-50.

3. Zygmunt A, Stanczyk J. Methods of evaluation of autonomic nervous system function. Arch Med Sci 2010; 6: 11-8.

4. Wolf MM, Varigos GA, Hunt D, Sloman JG. Sinus arrhythmia in acute myocardial infarction. Med J Australia 1978; 2: 52-3.

5. Olasinska-Wisniewska A, Mularek-Kubzdela T, Seniuk W, et al. Prognostic value of decreased heart rate variability in long term follow-up in patients with acute myocardial infarrction treated with thrombolysis. Arch Med Sci 2009; 5: 97-102.

6. Kleiger RE, Miller JP, Bigger JT Jr, et al. Decreased heart rate variability and its association with increased mortality after acute myocardial infarction. Am J Cardiol 1987; 59: 256-62.
7. Nolan J, Batin PD, Anders R, et al. Prospective study of heart rate variability and mortality in chronic heart failure: results of the United Kingdom heart failure evaluation and assessment of risk trial (UK- HEART). Circulation 1998; 98: 1510-16.

8. Buchhorn R, Hammersen A, Bartmus D, Bursch J. The pathogenesis of heart failure in infants with congenital heart disease. Cardiol Young 2001; 11: 498-504.

9. Massin M, von Bernuth G. Clinical and haemodynamic correlates of heart rate variability in children with congenital heart disease. Eur J Pediatr 1998; 157: 967-71.

10. Heragu NP, Scott WA. Heart rate variability in healthy children and in those with congenital heart disease both before and after operation. Am J Cardiol 1999; 83: 1654-57.

11. Kucharska W, Maslowska E, Wojcik E, et al. Heart rate variability in children with selected congenital heart disease. Folia Cardiol 2004; 11: 39-45.

12. Bobkowski W, Siwinska A, Zachwieja J, et al. Relationship between spectral parameters of heart rate variability and ventricular arrhythmias in children with mitral valve prolapse. Folia Cardiol 2003; 3: 373-79.

13. Jung J, Heisel A, Tscholl D, et al. Factors influencing heart rate variability in patients with severe aortic valve disease. Clin Cardiol 1997; 20: 341-4.

14. Stein KM, Borer JS, Hochreiter C, et al. Prognostic value and physiological correlates of heart rate variability in chronic severe mitral regurgitation. Circulation 1993; 88: 127-35.

15. Fauchier L, Babuty D, Cosnay P, et al. Prognostic value of heart rate variability for sudden death and major arrhythmic events in patients with idiopathic dilated cardiomiopathy. J Am Coll Cardiol 1999; 33: 1203-7.

16. Alter P, Grimm W, Vollrath A, et al. Heart rate variability in patients with cardiac hypertrophy - relation to left ventricular mass and etiology. Am Heart J 2006; 151 : 829-36.

17. Carney RM, Blumenthal JA, Freedland KE, et al. Low heart variability and the effect of depression on post-myocardial infarction mortality. Arch Intern Med 2005; 165 : 1486-91.

18. Karcz M, Ruzyllo W. Heart rate variability in dilated cardionyopathy. Folia Cardiol 2001; 8: 85-90.

19. Butera G, Bonnet J, Kachaner J, et al. Heart rate variability in children with hypertrophic cardiomyopathy. Heart 2003; 89: 205-6.

20. Chakko S, Mulingtapang RF, Huikuri HV, et al. Alternations in heart rate variability and its circadian rhythm in hypertensive patients with left ventricular hypertrophy free of coronary artery disease. Am Heart J 1993; 126: 1364-72.

21. Mandwat MK, Wallbridge DR, Pringle SD, et al. Heart rate variability in left ventricular hypertrophy. Br Heart J 1995; 73: 139-44.

22. Perkiomaki JS, Markku J, Ikaheimo SM. Dispersion of the QT interval and autonomic modulation of heart rate in hypertensive men with and without left ventricular hypertrophy. Hypertension 1996; 28: 16-9.

23. Hoffman J, Grimm W, Menz V. Heart rate variability and major arrhythmic events in patients with idiopathic dilated cardiomyopathy. PACE 1996; 1841-44.

24. Myerburg JM. Sudden cardiac death in persons with normal (or near normal) hearts. Am J Cardiol 1977; 79: 3-9.

25. Saul JP, Arai Y, Berger RD, et al. Assessment of autonomic regulation in chronic congestive heart failure by heart rate spectral analysis. Am J Cardiol 1988; 61: 1292-9. 